\title{
Migraine in children and adults born preterm : A nationwide register linkage study
}

\section{Strang-Karlsson, Sonja}

2021-05

Strang-Karlsson , S, Alenius , S, Näsänen-Gilmore , P , Nurhonen , M , Haaramo , P , Evensen , K A I , Vääräsmäki , M , Gissler , M , Hovi , P \& Kajantie , E 2021 , ' Migraine in children and adults born preterm : A nationwide register linkage study ', Cephalalgia, vol. 41 , no. 6 , pp. 677-689 . https://doi.org/10.1177/0333102420978357

http://hdl.handle.net/10138/331814

https://doi.org/10.1177/0333102420978357

cc_by

publishedVersion

Downloaded from Helda, University of Helsinki institutional repository.

This is an electronic reprint of the original article.

This reprint may differ from the original in pagination and typographic detail.

Please cite the original version. 


\title{
Migraine in children and adults born preterm: A nationwide register linkage study
}

Cephalalgia

202I, Vol. 4I(6) 677-689

(C) International Headache Society 2020

Article reuse guidelines:

sagepub.com/journals-permissions

DOI: $10.1177 / 0333102420978357$

journals.sagepub.com/home/cep

@SAGE

\author{
Sonja Strang-Karlsson ${ }^{1,2,3}$ (D), Suvi Alenius ${ }^{1,3}$, \\ Pieta Näsänen-Gilmore ${ }^{1,4}$, Markku Nurhonen', \\ Peija Haaramo', Kari Anne I Evensen ${ }^{5,6,7,8}$, Marja Vääräsmäki', \\ Mika Gissler ${ }^{1,10}$, Petteri Hovi ${ }^{1,3}$ and Eero Kajantie ${ }^{1,3,5,9}$
}

\begin{abstract}
Objective: Being born preterm is related to adverse health effects later in life. We studied whether preterm birth predicts the risk of migraine.

Methods: In this nationwide register study, we linked data from six administrative registers for all 235,624 children liveborn in Finland (January 1987 to September 1990) and recorded in the Finnish Medical Birth Register. $\mathrm{n}=228,610$ (97.0\%) had adequate data and were included. Migraine served as primary outcome variable and was stringently defined as a diagnosis from specialised health care and/or $\geq 2$ reimbursed purchases of triptans. We applied sex- and birth yearstratified Cox proportional hazard regression models to compute hazard ratios and confidence intervals (95\% confidence intervals) for the association between preterm categories and migraine. The cohort was followed up until an average age of 25.1 years (range: $23.3-27.0$ ).

Results: Among individuals born extremely preterm (23-27 completed weeks of gestation), the adjusted hazard ratios for migraine was 0.55 (0.25-1.24) when compared with the full-term reference group (39-4I weeks). The corresponding adjusted hazard ratios and $95 \%$ confidence intervals for the other preterm categories were: Very preterm (28-3I weeks); 0.95 (0.68-I.3I), moderately preterm (32-33 weeks); 0.96 (0.73-I.27), late preterm (34-36 weeks); I.0I (0.9I-I.II), early term (37-38 weeks); 0.98 (0.93-I.03), and post term (42 weeks); 0.98 (0.89-I.08). Migraine was predicted by parental migraine, lower socioeconomic position, maternal hypertensive disorder and maternal smoking during pregnancy.
\end{abstract}

Conclusion: We found no evidence for a higher risk of migraine among individuals born preterm.

\section{Keywords}

Neonatal, gestational age, cohort studies, epidemiology, adult outcomes, prematurity

Date received: 5 June 2020; revised: 7 September 2020; 25 October 2020; accepted: 12 November 2020

\footnotetext{
'Finnish Institute for Health and Welfare, Helsinki, Finland

${ }^{2}$ Department of Clinical Genetics, HUSLAB, University of Helsinki and Helsinki University Hospital, Helsinki, Finland

${ }^{3}$ Children's Hospital, University of Helsinki and Helsinki University Hospital, Helsinki, Finland

${ }^{4}$ Global Health group, University of Tampere, Tampere, Finland

${ }^{5}$ Department of Clinical and Molecular Medicine, Norwegian University of Science and Technology, Trondheim, Norway

${ }^{6}$ Department of Public Health and Nursing, Norwegian University of Science and Technology, Trondheim, Norway
}

\footnotetext{
${ }^{7}$ Unit for Physiotherapy Services, Trondheim Municipality, Trondheim, Norway

${ }^{8}$ Department of Physiotherapy, Oslo Metropolitan University, Oslo, Norway

${ }^{9}$ Faculty of Medicine, PEDEGO Research Unit, MRC Oulu, Oulu

University Hospital and University of Oulu, Oulu, Finland

${ }^{10}$ Karolinska Institute, Department of Neurobiology, Care Sciences and

Society, Stockholm, Sweden
}

Corresponding author:

Sonja Strang-Karlsson, Department of Clinical Genetics, HUSLAB, University of Helsinki and Helsinki University Hospital, PO Box 160, 00029 Helsinki, Finland.

Email: sonja.strang@helsinki.fi 


\section{Introduction}

One in ten children worldwide is born preterm, before 37 completed weeks of gestation (1). Together with the increased survival rates among preterm infants, this translates into a substantial share of preterm individuals within the adult population. Preterm birth confers an increased risk of neurocognitive impairment (2), mental health problems (3), elevated blood pressure and insulin resistance $(4,5)$ and, at least in populations born from the 1970s onwards, ischemic heart disease and stroke (6-9).

During the past decades there has been a surge in studies elucidating the effects of early life events on adult health. Still, studies on migraine in individuals born preterm are sparse, and little is known about the possible effects of prematurity on the development of migraine. Babies born preterm are deprived of the last weeks or months of intrauterine life, a period of rapid development of the vulnerable foetal brain. Additionally, they often face immaturity-related illnesses and complications postnatally. Whether these neurodevelopmental risk factors put them at risk for migraine later in life is unknown.

Migraine is linked with risk of stroke and cardiovascular disease (10), gestational hypertension (11), psychiatric comorbidity (12), low socioeconomic position (13), and adverse pregnancy-related outcomes $(11,14)$ factors that have all been linked with prematurity as well $(3,6,7,15,16)$. Given that migraine and prematurity share a number of associated conditions and knowing that prematurity affects numerous aspects of neurological development (17), we set out to study whether preterm birth, across the entire gestational age range, is associated with migraine in children or young adults.

\section{Methods}

\section{Data collection}

We conducted a population-based register linkage study to investigate the occurrence of migraine across different gestational age groups. We utilised data from multiple national administrative registers and linked these data together, using an encrypted form of the unique personal identifier that is assigned to all residents in Finland and remains the same throughout life.

The six nationwide registers were: i) the Finnish Medical Birth Register (MBR), ii) the Central Population Register (CPR), iii) the Education Register maintained by Statistics Finland, iv) the Register of Congenital Malformations, v) the Finnish Care Register for Health Care, formerly the Hospital Discharge Register, and vi) the Finnish Prescription Register. The registers, the validity of the data, and the study design have been described in detail previously (18-22).

We utilised the MBR for obtaining data on maternal background characteristics, pregnancy, delivery and perinatal outcomes, and for defining the study cohort. The MBR covers all children born in Finland from 1987 onwards. Data on death, emigration and family relationships came from the CPR (updated through April 2012) and data on educational achievements from the Education Register (updated through December 2012). Data on congenital anomalies came from the Register of Congenital Malformations (January 2015). The Finnish Care Register for Health Care contains data on all inpatient hospital discharges since 1967, and since 1998 also on outpatient hospital visits in public hospitals, providing us with data on migraine diagnoses from the specialised health care (data extracted December 2015). Diagnoses (one main and two secondary diagnoses) are recorded in the register using the International Statistical Classification of Diseases and Related Health Problems, 8th revision (ICD-8) during the years 1969-1986, ICD-9 during 1987-1995, and ICD-10 from 1996 onwards (23). We obtained data on triptan purchases from the Finnish Prescription Register, maintained by the Finnish Social Insurance Institution (data extracted December 2013). In this register, all reimbursed prescription drug purchases are documented since 1994, with 97\% coverage during the first decade (22). The reimbursement system covers all permanent residents in Finland living in a non-institutional setting. Thus, reimbursed purchases of drugs prescribed at public health services, private practices as well as occupational health are covered. Triptans were launched in 1991 and entered the market in Finland in 1992. In 2003, the first triptan was labelled for use in children $\geq 12$ years in Finland, although paediatric off-label use was widespread even before that (22). Throughout the study period, triptans were prescriptions drugs in Finland, unapproved for over-the-counter sale.

\section{Standard protocol approvals, registrations, and patient consents}

The study was approved by the local ethics committee, The Finnish Ministry of Health and Social Affairs, and relevant register authorities. Based on EU and Finnish legislation, individual consents are not required in this study type based on pseudonymised register data.

\section{Study cohort}

The study cohort comprises all live-born children in Finland between 1 January 1987 and 30 September 1990 who were recorded in the nationwide MBR 
$(\mathrm{n}=235,624,99.8 \%$ of live-born children) (Figure 1). We excluded participants as described in Figure 1 in order to keep the exposure as accurate as possible: Those with missing or doubtful gestational age or birth weight standard deviation (SD) score, and those with at least one major congenital anomaly. Six outliers diagnosed with migraine before the age of 4 years were omitted, since diagnosing migraine in toddlers unable to verbally describe symptoms is challenging and none of them had a migraine diagnosis or triptan purchase after age 4, indicating probable reporting error. The final sample included in all analyses consists of 228,610 participants $(97.0 \%$ of the original birth cohort).

\section{Exposure}

Gestational age at birth served as exposure variable. Estimation of gestational age was based on the last menstrual period and ultrasound estimation (when available). The gestational age categories were as follows: Extremely preterm, 23-27 completed weeks $(\mathrm{n}=522,0.2 \%)$; very preterm, 28-31 weeks $(\mathrm{n}=982$, $0.4 \%)$; moderately preterm, $32-33$ weeks $(n=1207$, $0.5 \%)$; late preterm, 34-36 weeks $(\mathrm{n}=8516,3.7 \%)$; early term, $37-38$ weeks $(n=40,570,17.7 \%)$; full term, 39-41 weeks $(\mathrm{n}=167,646,73.3 \% \quad$ [reference group]), and post term, 42 weeks $(\mathrm{n}=9173,4.0 \%)$ $(24,25)$.

\section{Outcome variables}

Our primary outcome variable was a diagnosis of migraine from specialised health care and/or at least two reimbursed purchases of triptans. As secondary outcomes, we analysed diagnoses of migraine and triptan purchases separately. Specialised health care diagnoses were extracted from the Finnish Care Register for Health Care as described above. The ICD codes extracted are shown in Table 1. As the register does not cover primary health care, we extracted diagnoses only from specialised health care. To capture migraineurs diagnosed in the primary care setting, we used purchases of triptans as a proxy for migraine diagnoses. The cut-off was set to $\geq 2$ reimbursed purchases, in order to increase specificity and avoid classifying patients trying triptans on a single occasion as migraineurs. In Finland, reimbursed medication can be bought for no longer than 3 months at a time.

For the primary outcome, we defined age at event as the age at first diagnosis, or age at the first purchase (of $\geq 2$ purchases) of triptans, whichever occurred first.

\section{Statistical analyses}

We applied Cox proportional hazard regression models to compute hazard ratios (HRs) and $95 \%$ confidence intervals (CIs) for the association between gestational age categories and migraine. The term-born group (3941 weeks) served as the reference group. The models were stratified by birth year and sex of the study

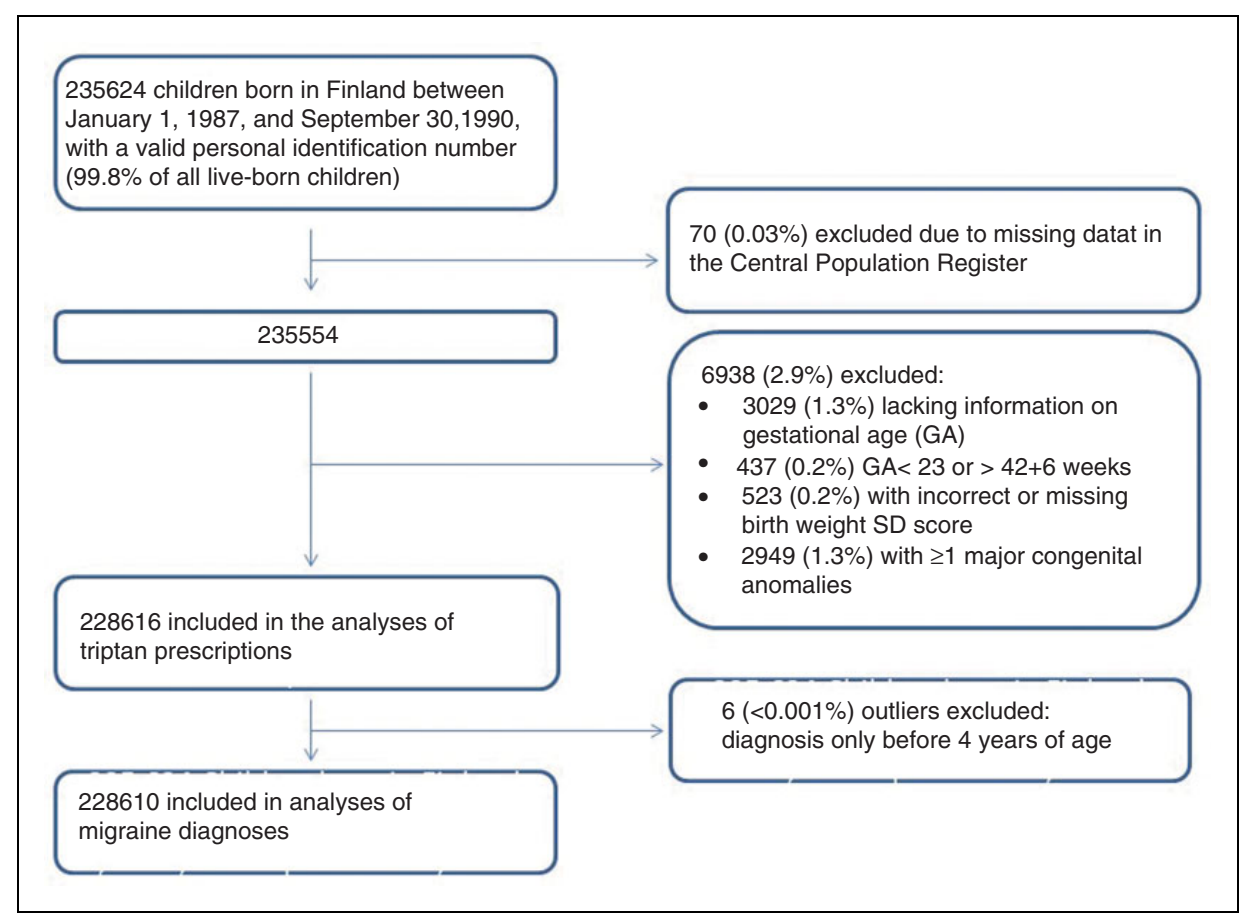

Figure I. Study cohort. 
Table I. Summary of ICD-8, ICD-9, and ICD-I0 codes extracted and included in the analyses.

\begin{tabular}{|c|c|c|c|}
\hline ICD-8 & ICD-9 & ICD-I0 & Diagnosis \\
\hline- & 346 & G43 & Migraine \\
\hline- & 346 IA & G43.0 & Migraine without aura (common migraine) \\
\hline- & $3460 \mathrm{~A}$ & G43.I & Migraine with aura (classical migraine) \\
\hline- & & & Including: \\
\hline- & - & G43.I & Migraine familial hemiplegic \\
\hline- & - & G43.I & Migraine with: Typical aura \\
\hline- & - & G43.I & Migraine with: Acute-onset aura \\
\hline- & - & G43.I & Migraine with: Prolonged aura \\
\hline- & - & G43.I & Migraine aura without headache \\
\hline- & - & G43.I & Migraine equivalents \\
\hline- & - & G43.I & Migraine basilar \\
\hline- & $3468 X$ & G43.2 & Status migrainosus \\
\hline- & $3468 X$ & G43.3 & Complicated migraine \\
\hline- & $3468 X$ & G43.8 & Other migraine \\
\hline- & - & & Including: \\
\hline- & - & G43.8 & Ophthalmoplegic migraine \\
\hline- & - & G43.8 & Retinal migraine \\
\hline 346.09 & $3469 X$ & G43.9 & Migraine, unspecified \\
\hline- & $3468 X$ & $\mathrm{G} 44.0^{\mathrm{a}}$ & Cluster headache syndrome ${ }^{a}$ \\
\hline- & - & & Including: \\
\hline- & - & G44.0 & Episodic cluster headache \\
\hline- & - & G44.0 & Paroxysmal hemicrania \\
\hline 346.01 & - & G44.0 & Horton's disease \\
\hline- & - & G44.0 & Migraine, episodic, chronic \\
\hline
\end{tabular}

${ }^{\text {a} G 44.0 ~ w a s ~ i n c l u d e d ~ i n ~ a n a l y s e s, ~ s i n c e ~ i t ~ e n c o m p a s s e s ~ t h e ~ s u b g r o u p ~ " m i g r a i n e, ~ e p i s o d i c, ~ c h r o n i c " ~ a n d ~ t h e ~ c o r r e s p o n d i n g ~}$ ICD-9 code $3468 \mathrm{X}$, which was also extracted, covers both migraine and cluster headache as well. In line with this reasoning, ICD-8 code 346.01 was also included.

ICD: International Statistical Classification of Diseases and Related Health Problems.

participants: Birth year, since it is expected to interfere with the likelihood of achieving a diagnosis, or triptan prescription (triptans were launched in 1991 and increased over the years), and sex because migraine prevalence and disease course differs between men and women, and in our cohort, the sex difference in migraine varied over time ( $p$-value for interaction between sex and time interval<0.01) $(22,26)$. The study participants were followed up until a) receiving their first diagnosis of migraine, b) until their first reimbursed purchase of triptans (in case the participant had $\geq 2$ purchases during follow-up), c) migration $(\mathrm{n}=3208,1.4 \%), \mathrm{d})$ death $(\mathrm{n}=2395,1.0 \%)$, or end of follow-up, whichever event occurred first. The follow-up (rounded to the closest 30 days) ended in December 2013 for variables including triptan purchases. The mean age at end of follow-up was 25.1 years (range: 23.3-27.0). In secondary analyses, when analysing diagnoses exclusively, the follow-up extended until December 2015, the mean age of the participants being 27.1 years (range: 25.3-29.0)

We adjusted for the following covariates, representing variables potentially associated with prematurity and migraine, and chosen on theoretical or statistical grounds: Birth weight SD score in relation to length of gestation (using Finnish reference values for intrauterine growth) (27), parental migraine (yes/no, either parent diagnosed in specialised health care between 1969-2015, not accounting for diagnoses before 1969 or parents possibly lost to follow-up), parental educational attainment (highest ever of either parent during follow-up) as a proxy for socioeconomic position, maternal age and marital status when giving birth (married, yes/no), birth order (first-born, yes/no), maternal hypertensive disorder (including preeclampsia and gestational hypertension) or gestational diabetes. Maternal smoking during pregnancy (yes/no) was derived from self-reports at the first antenatal clinic visit, usually at 9-10 completed gestational weeks. An adverse neonatal clinical course was operationalised in terms of an aggregate variable that included asphyxia, retinopathy of prematurity, or bronchopulmonary dysplasia (yes/no), and another aggregate variable called severe infant morbidity, including diagnoses of severe neurological, infectious, pulmonary or other neonatal morbidity. Both aggregate variables were described in detail previously (18). Additionally, we adjusted for a diagnosis of developmental delay or intellectual 
Table 2. Independent effects (unadjusted HRs, $95 \% \mathrm{Cl}$ ) of specific covariates on later risk of migraine (defined as a diagnosis of migraine from specialised health care, and/or $\geq 2$ reimbursed triptan purchases during follow-up).

\begin{tabular}{|c|c|c|c|c|}
\hline Covariate & $\mathrm{HR}$ & $95 \% \mathrm{Cl}$ & $p$-value & Migraine diagnosis, \\
\hline & & & & yes vs. no $n(\%)^{a}$ \\
\hline Female (versus male) & 3.12 & $2.99-3.26$ & $<0.001$ & $8136(7.3 \%)$ vs. 103,551 (92.7\%) \\
\hline Small for gestational age (birth weight SD score $\leq 2)^{b}$ & 1.12 & $1.00-1.25$ & 0.055 & $312(5.2 \%)$ vs. $5706(94.8 \%)$ \\
\hline Being first-born (yes) & 0.99 & $0.95-1.03$ & 0.59 & $4319(4.7 \%)$ vs. $86,728(95.3 \%)$ \\
\hline Maternal marital status at birth (married) ${ }^{c}$ & 0.97 & $0.92-1.01$ & 0.13 & $8536(4.8 \%)$ vs. $170,918(95.2 \%)$ \\
\hline Maternal smoking during pregnancy (yes) ${ }^{d}$ & 1.13 & $1.07-1.18$ & $<0.001$ & $1798(5.3 \%)$ vs. $32,393(94.7 \%)$ \\
\hline Gestational diabetes (yes) & 0.99 & $0.88-1.10$ & 0.78 & $339(4.7 \%)$ vs. $6888(95.3 \%)$ \\
\hline Maternal hypertensive disorder (yes) & 1.10 & $1.02-1.18$ & 0.018 & $728(5.2 \%)$ vs. $13,226(94.8 \%)$ \\
\hline Maternal age at birth (years) ${ }^{\mathrm{e}}$ & 0.99 & $0.98-0.99$ & $<0.001$ & $N / A^{f}$ \\
\hline Asphyxia, ROP, or BDP (yes) ${ }^{g}$ & 0.94 & $0.86-1.03$ & 0.18 & 526 (4.5\%) vs. II,288 (95.5\%) \\
\hline Severe infant morbidity (yes) ${ }^{h}$ & 0.95 & $0.78-1.16$ & 0.61 & 97 (4.3\%) vs. 2180 (95.7\%) \\
\hline \multicolumn{5}{|l|}{$\begin{array}{l}\text { Parental education, highest level } \\
\text { achieved by either parent }\end{array}$} \\
\hline Upper-secondary & \multicolumn{4}{|c|}{ Reference category } \\
\hline Post-secondary ${ }^{i}$ & 0.95 & $0.90-0.99$ & 0.020 & $2538(4.8 \%)$ vs. $50,378(95.2 \%)$ \\
\hline Lower tertiary $^{i}$ & 0.90 & $0.85-0.95$ & $<0.001$ & I33| (4.6\%) vs. 27,885 (95.4\%) \\
\hline Upper tertiary or more ${ }^{i}$ & 0.79 & $0.75-0.84$ & $<0.001$ & I 463 (4.0\%) vs. 35,049 (96.0\%) \\
\hline Basic or unknown ${ }^{i}$ & 1.07 & $0.99-1.16$ & 0.11 & 698 (5.3\%) vs. 12,421 (94.7\%) \\
\hline Intellectual or developmental disability ${ }^{j}$ & 0.81 & $0.6 \mathrm{I}-1.07$ & 0.14 & 50 (3.8\%) vs. $1274(96.2 \%)$ \\
\hline Parental migraine $e^{k}$ & 2.07 & $1.94-2.22$ & $<0.001$ & $930(9.2 \%)$ vs. $9232(90.8 \%)$ \\
\hline
\end{tabular}

Note: Univariate Cox regression analyses were fit. The cohort $(n=228,610)$ was followed up until December 20I3. Cases who emigrated or died before end of follow-up were censored.

${ }^{a}$ Refers to the share $(n, \%)$ of individuals with versus without a migraine diagnosis within the respective covariate category. Crude values shown: Cases who emigrated or died before end of follow-up not accounted for.

beference 27.

Missing values: ${ }^{\mathrm{C}} 105 \mathrm{I}(0.5 \%),{ }^{\mathrm{d}} 4426$ (1.9\%), ${ }^{\mathrm{e}} 3(<0.001 \%)$.

${ }^{f}$ Continuous variable.

${ }^{8}$ Diagnosed with asphyxia, retinopathy of prematurity (ROP) or bronchopulmonary dysplasia (BPD) before 3 months of age.

${ }^{\mathrm{h}}$ Aggregate variable including diagnoses of severe neurological, infectious, pulmonary or other morbidity in the neonatal period (I8).

'Compared with reference category (upper-secondary).

'Diagnosed with developmental delay and/or intellectual disability (ICD-I0 codes F84, F70-F79; ICD-9 codes 2990, 2998, 2999, 3I7-3I9) by December 2012 (49).

${ }^{\mathrm{k}}$ Either biological parent diagnosed with migraine in special health care between 1969-2015 (parents who died or migrated not censored, and those who received a diagnosis before 1969 not accounted for).

HR: Hazard ratio; Cl: confidence interval.

disability. Table 2 shows the unadjusted associations between the individual covariates and migraine.

Analyses were run with and without adjustments for covariates, and finally repeated with singletons only. Missing values were treated as missing, without data imputation, meaning that cases with missing values with respect to any covariate were omitted from the model (a maximum of 5085 [2.2\%] cases were excluded from the analyses due to missing data). In adjusted models, covariates were introduced in clusters. Potential interactions between gestational age and sex or gestational age and foetal growth (for which small for gestational age [SGA] served as a proxy) were examined by introducing the corresponding interaction terms in the models. SGA was defined as a birth weight $\leq 2$ SD score for gestational age.

We checked the validity of the proportional hazards assumption using the Schoenfeld residuals-based test in
R package survival (28) and found no time-dependent HRs for the gestational age categories and no serious time dependencies among the covariate effects that would distort the results obtained from the proportional hazards regression. Otherwise, analyses were conducted with SPSS version 25 (IBM SPSS Statistics; IBM Corporation). A two-sided $p$-value of $<0.05$ was considered significant.

\section{Data availability}

All relevant analysis results are shared and published in this article. No individual-level data is openly available: Only members of the study groups were granted access to the sensitive data from the relevant registers and further access rights are subject to permission from the registers. Interested researchers may apply for access rights from the registers (18). 


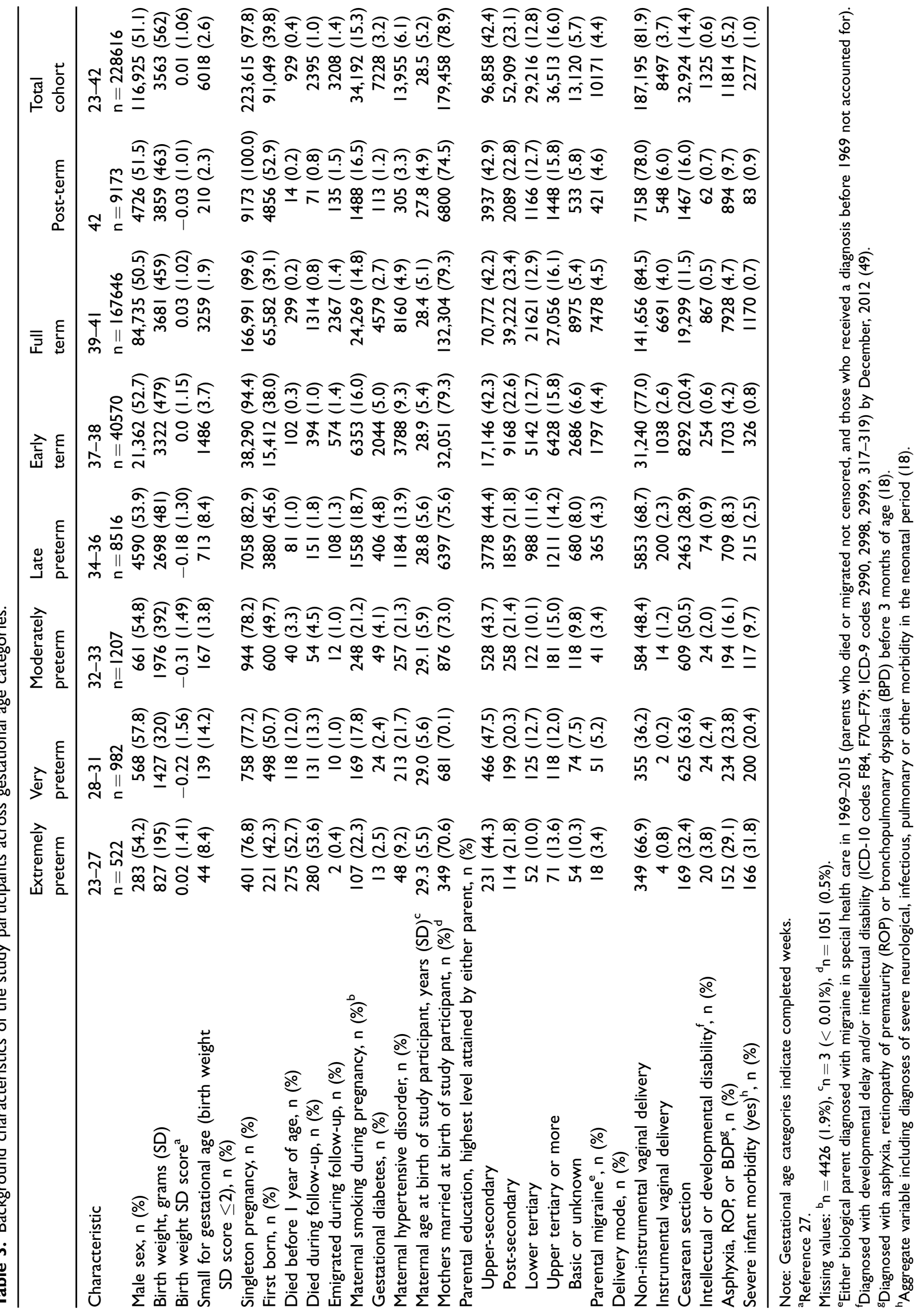




\section{Results}

Table 3 shows background characteristics of the study cohort by gestational age at birth. As expected, adverse pregnancy and neonatal characteristics were more common among those born preterm as opposed to full term ( $p$-values $<0.05$, chi-squared test or t-test). Indicators of socioeconomic position were less advantageous among preterm individuals (lower parental educational attainment, fewer mothers married at birth of study participant, $p$-values $<0.05$, chisquared test). Rates of parental migraine were similar between groups ( $p$-value 0.25 , chi-squared test).

\section{Univariate association between covariates and migraine}

Table 2 shows that risk of migraine was higher in women and among those whose parents suffered from migraine. Smoking during pregnancy, maternal hypertensive disorder, and lower parental educational attainment was associated with higher risk of migraine. Higher maternal age at birth was linked with lower risk of migraine in the offspring; however, this association turned non-significant when analyses were confined to the interquartile range (ages 24-32 years). The association between being SGA and higher risk of migraine only bordered on significance (HR 1.12, 1.00 to 1.25). Higher birth weight SD score was associated with lower risk of migraine among those born full term only (HR per SD 0.97, 0.95-0.99), but the direction of the associations in the other gestational age categories varied and were statistically insignificant. Univariate analyses showed no associations between other neonatal morbidity covariates and subsequent migraine.

\section{Distribution of migraine according to sex and age at onset}

During the follow-up period, the cumulative incidence of migraine was higher among females than males $(7.3 \%$ vs. $2.4 \%$ for the main outcome variable, $p<0.01$, chi-squared test) (Table 4). The sex difference was observable also when analysing secondary outcomes; that is, migraine diagnoses, migraine with aura, and triptan purchases separately, being most marked for triptan purchases $(5.1 \%$ vs. $1.0 \%$, $p$-value $<0.01$, chi-squared test) (Table 4). The age at onset of migraine varied according to sex, as shown in Figure 2. Females were on average older than males at onset of migraine and had a unimodal age at onset-distribution (medians 18.9 vs. 15.4 years for females and males, $p$-value $<0.01$, Mann-Whitney Utest). A larger proportion of male migraineurs, compared to female migraineurs, had onset of migraine below the age of 15 years $(46.2 \%$ vs $18.5 \%, p<0.01)$. The age at onset of migraine among males was more evenly, slightly bimodally, distributed (interquartile range for males and females: 12.3-20.2 and 16.0-21.7 years). At the end of follow-up, in fully adjusted logistic regression analyses and when we excluded cases who were lost to follow-up due to death or migration, female sex conferred a threefold overall risk of migraine (adjusted odds ratio 3.21, 3.07-3.35).

\section{Gestational age and later migraine}

On crude group comparisons (Table 4), migraine appeared less common among those born at the lowest gestational age. However, mortality among these extremely preterm children was high, causing loss to follow-up at early ages before possibly developing migraine, and thus the follow-up time needs to be accounted for in analyses. In order to do so, Cox regression models, accounting for the follow-up time for each participant, were fitted. As shown in Table 5 and Figure 3, adjustment for covariates listed in Table 5 revealed no significant association between preterm birth and subsequent migraine. The results remained unchanged after an exclusion of multiple births. There were no significant interactions between gestational age categories and sex, meaning that the effect of prematurity on later migraine did not differ between males and females.

In the final model (Table 5), the following covariates predicted the primary outcome (i.e. a migraine diagnosis and/or $\geq 2$ triptan purchases) in fully adjusted models: Parental migraine (aHR 2.07, 95\% CI: 1.93 2.21), maternal smoking during pregnancy (aHR 1.08, 1.02-1.14), maternal hypertensive disorder (aHR 1.13, 1.04-1.22), maternal age at birth (aHR per year 0.99, 0.98-0.99), being first born (aHR 0.94, 0.90-0.98), and parental educational attainment (aHR 0.83, 0.78-0.89; for upper tertiary education or more compared to upper secondary). SGA was not associated with later migraine.

\section{Discussion}

We report on a well-characterised nationwide population-based study exploring the association between prematurity across the entire gestational age range and subsequent risk of migraine up to adulthood. By linking different national registers and using stringent criteria to define migraine, we were able to identify 10,922 migraineurs among 228,610 study participants in the birth cohort. We found no evidence for an association between preterm birth and later migraine, triptan purchases or migraine with aura. Parental migraine, lower socioeconomic position, maternal 
Table 4. Distribution of migraine outcome variables at end of follow-up (not accounting for length of follow-up or those lost to follow-up due to death or migration).

\begin{tabular}{|c|c|c|c|c|}
\hline \multirow[b]{2}{*}{ Gestational age category, weeks } & \multicolumn{4}{|c|}{ Outcome variable, cases (\%) } \\
\hline & $\begin{array}{l}\text { Migraine diagnosis } \\
\text { and/or } \geq 2 \text { triptan } \\
\text { purchases }^{\mathrm{a}}\end{array}$ & $\begin{array}{l}\text { Migraine } \\
\text { diagnosis }^{b}\end{array}$ & $\begin{array}{l}\geq 2 \text { triptan } \\
\text { purchases }^{\mathrm{a}}\end{array}$ & $\begin{array}{l}\text { Diagnosis of } \\
\text { migraine with } \\
\text { aura ever,c }\end{array}$ \\
\hline Number in analyses & $\mathrm{n}=228610^{\mathrm{d}}$ & $\mathrm{n}=228610^{\mathrm{d}}$ & $\mathrm{n}=228616^{\mathrm{d}}$ & $\mathrm{n}=228610^{\mathrm{d}}$ \\
\hline $\begin{array}{l}\text { Extremely preterm, 23-27 } \\
\qquad(\mathrm{n}=522)\end{array}$ & $6(1.1)$ & $3(0.6)$ & $5(1.0)$ & $0(0.0)$ \\
\hline $\begin{array}{l}\text { Very preterm, 28-3I } \\
\qquad(\mathrm{n}=982)\end{array}$ & $38(3.9)$ & $26(2.6)$ & $18(1.8)$ & $6(0.6)$ \\
\hline $\begin{array}{l}\text { Moderately preterm, } 32-33 \\
\quad(n=1206)\end{array}$ & $52(4.3)$ & $31(2.6)$ & $38(3.1)$ & $10(0.8)$ \\
\hline $\begin{array}{l}\text { Late preterm, } 34-36 \\
\qquad(\mathrm{n}=8516)\end{array}$ & $405(4.8)$ & $269(3.2)$ & $238(2.8)$ & $78(0.9)$ \\
\hline $\begin{array}{l}\text { Early term, 37-38 } \\
\quad(n=40,569)\end{array}$ & 1895 (4.7) & $1252(3.1)$ & $1183(2.9)$ & $414(1.0)$ \\
\hline $\begin{array}{l}\text { Full term, 39-4I } \\
\qquad(n=167642)\end{array}$ & $8095(4.8)$ & $5257(3.1)$ & $5125(3.1)$ & 1765 (I.I) \\
\hline $\begin{array}{r}\text { Post term, } 42 \\
(\mathrm{n}=9173)\end{array}$ & $431(4.7)$ & $285(3.1)$ & $263(2.9)$ & $95(1.0)$ \\
\hline Total cohort ${ }^{d}$ & $10,922(4.8)$ & $7123(3.1)$ & $6870(3.0)$ & $2368(1.0)$ \\
\hline Men $(n=116,923)$ & $2786(2.4)$ & $2149(1.8)$ & $1218(1.0)$ & $619(0.5)$ \\
\hline Women $(n=111,687)$ & $8136(7.3)$ & $4974(4.5)$ & $5652(5.1)$ & $1749(1.6)$ \\
\hline
\end{tabular}

Note: Gestational age categories indicate completed weeks.

${ }^{\mathrm{a}}$ For outcome variables including triptan purchases, follow-up ended in December 2013 (mean age 25.I years; range: 23.3-27.0).

${ }^{\mathrm{b}}$ For outcomes on diagnoses only, the follow-up ended in December 2015 (mean age 27.I years; range: 25.3-29.0).

'Participants who had ever received a diagnosis of migraine with aura (codes 3460A or G43.I, from the ICD-9 and ICD-I0, respectively).

${ }^{d}$ Outcomes including diagnoses covered $n=228,610$ participants due to six excluded outliers as described in Figure I. For outcomes including triptan purchases only, $n=228,616$.

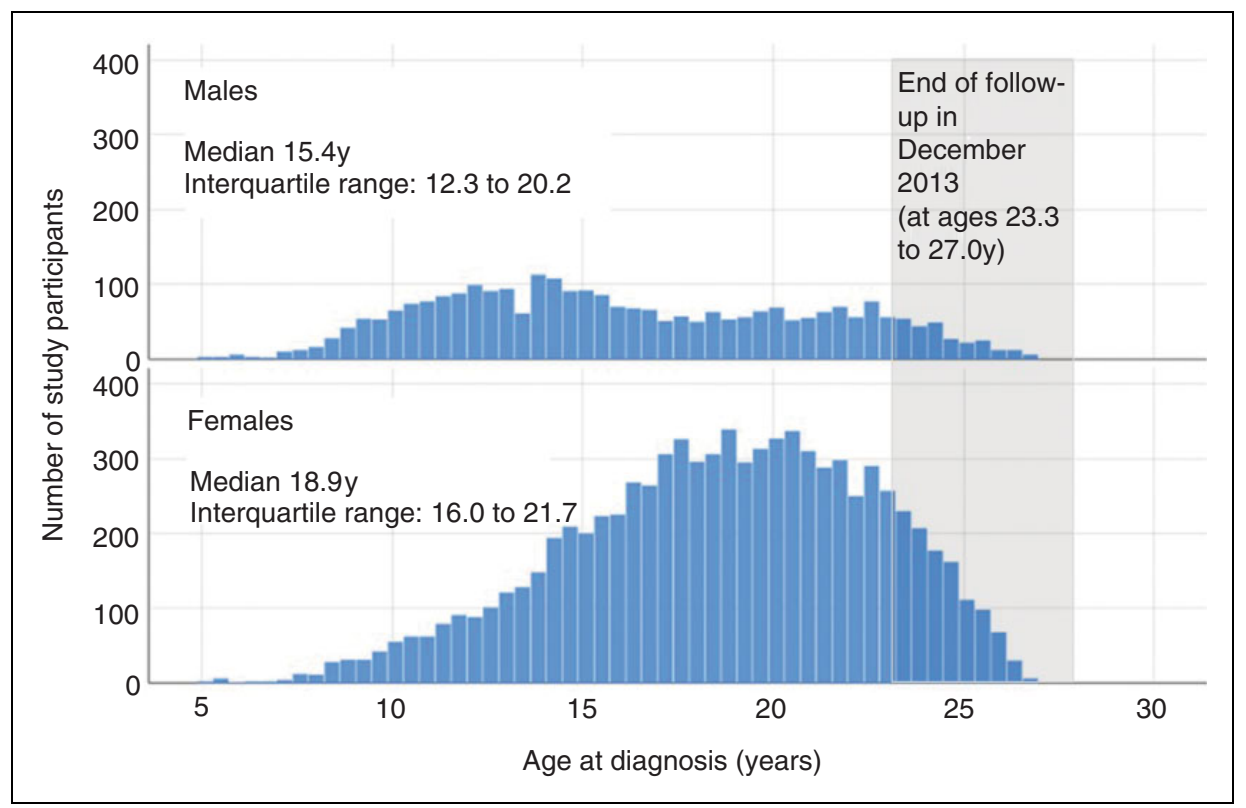

Figure 2. Age distribution at onset of migraine among males and females. 


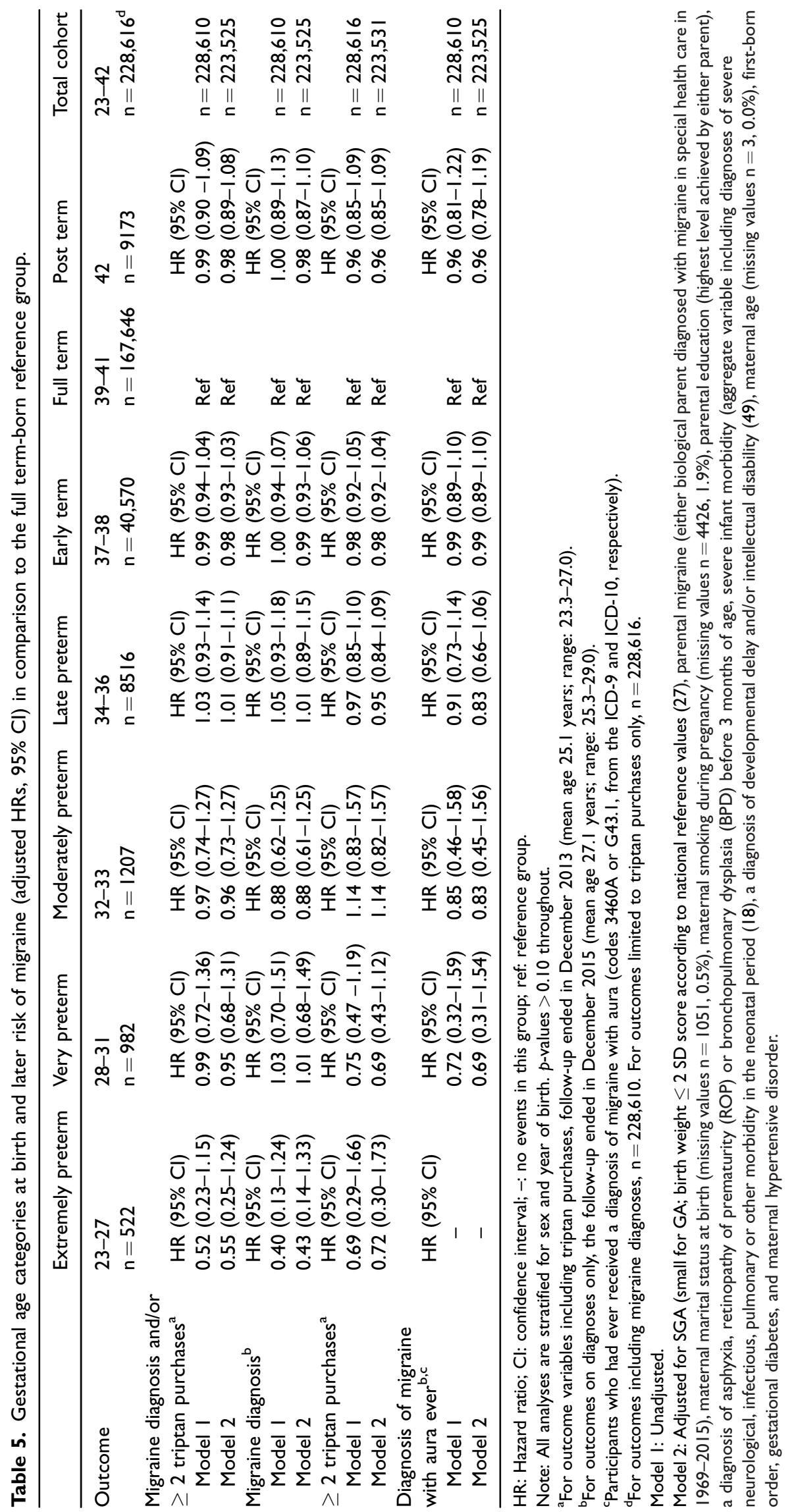




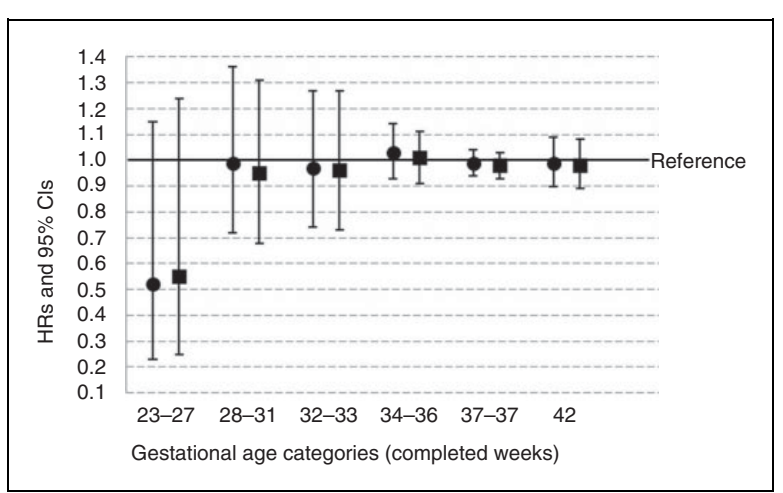

Figure 3. Risk of migraine according to gestational age category. Note: Migraine was defined as a migraine diagnosis and/or $\geq 2$ triptan purchases. The study participants were followed up until a mean age of 25.I years (range 23.3-27.0). The term born group (39-4I completed gestational weeks) served as the reference group, indicated by the thick horizontal line $(H R=1.0)$. Closed circles represent unadjusted $\mathrm{HRs}$, and closed square represent fully adjusted HRs corresponding to Model 2 in Table 5. Whiskers indicate the $95 \% \mathrm{Cls}$.

hypertensive disorder and - interestingly - also maternal smoking during pregnancy, predicted higher risk of migraine in the offspring.

Only a handful of previous studies have explored preand perinatal predictors of headache in general and migraine in particular. A study on mandibular function in preterm children and term born controls aged 8-10 years $(n=114)$ showed no group difference in selfreported headache (29). Also in line with our finding, another study on maternal-reported headache (migraine not studied) in 7-11-year-old children failed to report any effects of gestational age (30). In a retrospective chart review of a clinical sample of paediatric migraineurs, a history of care in neonatal intensive care units (NICU) predicted higher levels of pain medication for migraine and an earlier age at onset of migraine in unadjusted analyses (31). Prematurity was not separately investigated. The authors speculated that pain experiences in the NICU might alter later pain experiences through neuronal plasticity and the resulting central hyperexcitability. In yet another retrospective chart review of a clinical sample of 190 paediatric headache patients, a history of birth asphyxia was more common in the migraine than the tension-type headache group (32); however, the study was limited by lack of a nonheadache control group, lack of adjustment for confounders, and the definition of asphyxia was partly based on recall. These findings are not in line with ours, since in the current study, neonatal morbidity variables did not predict later migraine.

In a questionnaire-based study on 7354 adults aged 19-41 years, foetal growth retardation, operationalised in terms of a birth weight for gestational age below the third percentile (corresponding approximately to $-2 \mathrm{SD}$ used in our study) was associated with increased odds for migraine among males only (33). However, the study was limited by lack of adjustment for confounders and the effect of prematurity was not explored, thus it remains unclear whether the effect of SGA was attributable to lower gestational age, given that SGA individuals usually are overrepresented in the lower gestational age groups. In contrast, another study on 11-year-old children born at term showed no association between being born SGA and migraine or tension-type headache (34); however, their criterion for SGA (birth weight for gestational age $<10$ th percentile) was less strict and migraine epidemiology in children differs from that after puberty (35), possibly partly explaining the discrepancy in results between the two aforementioned studies. These two studies, together with the lack of a clear-cut link between SGA and later migraine in our study, suggest there is insufficient evidence to date for a link between foetal growth retardation and later migraine.

In the current study, maternal smoking during pregnancy was associated with an increase in risk of migraine (HR 1.08) in the offspring, despite adjustment for confounders. Although a causal association cannot be verified in a register-study setting, we consider this finding interesting and potentially of great importance, since maternal smoking during pregnancy is an actionable risk factor and prenatal nicotine exposure has detrimental effects on the developing foetal brain (36). Our finding is partly paralleled by a retrospective case-controlled $(n=1440)$ study based on interviews and standardised questionnaires, showing that prenatal smoking exposure increased the risk of chronic daily headache but not episodic headache among 5-12-year-olds (37). As discussed by the authors, the study had limited ability to adjust for socioeconomic position. Our finding is also partly in line with two longitudinally studied Brazilian birth cohorts showing higher rates of maternal-reported headache (migraine not studied) in 7-11-year-olds whose mothers smoked during pregnancy (30). Another study reported an association between maternal smoking during pregnancy and tension-type headache in 11-year-old children; yet, no association was seen in adjusted analyses or with regard to migraine (34). This discrepancy in results in comparison with our study may, again, be related to the age range, since the study in question was conducted in childhood before the peak prevalence of migraine (26).

The sex differences in the present study as well as the link between lower socioeconomic position and risk of migraine were as expected based on previous research $(13,15,26,35)$. Likewise, parental migraine predicted a higher risk of migraine in the offspring, unsurprisingly, considering the genetic components of migraine $(38,39)$. The association between maternal hypertensive disorder and increased risk of migraine in the offspring 
in the current study is interesting but needs replication. Although gestational hypertensive disorders are more common among migraineurs according to some studies (11), it is not recognised as an established risk factor for offspring migraine.

Even though migraine is primarily an episodic condition, it may progress into chronic migraine, which has features in common with other chronic pain disorders (40). Studies have linked migraine to musculoskeletal symptoms (41) and dysfunctions (42). However, there is no clear association between gestational age and musculoskeletal pain: One cohort study reported an association between preterm birth and chronic pain (43), but other larger studies found no association between preterm birth and chronic pain in adolescents (44) or widespread musculoskeletal pain in young adults (45). With this background, the lack of association between gestational age and migraine in our study may not be unexpected.

The strengths of the study include the sizeable national birth cohort with high coverage and follow-up into adulthood. The whole gestational age range is represented, which allows for analyses not only across the whole spectrum of prematurity, but also across early term and post term birth categories. The outcome variables were derived from nationwide registers with proven high validity $(18,22)$. Linkage between several national registers further enabled us to adjust for possible confounders and explore the effect of background variables with negligible numbers of missing values. The ethnically homogenous population of Finland at the time when the study participants were born, with only $0.8 \%$ of residents having a foreign background, presumably reduces possible confounding by genetic factors (46). On the other hand, it also diminishes the generalisability of the results to more ethnically diverse populations, which is a limitation.

As to limitations, our study does not provide data on the lifetime risk of migraine as the follow-up ended before 30 years of age, a decade prior to the peak prevalence of migraine (47). Nevertheless, at least up until young adulthood, we detected no association between gestational age and migraine. Future studies with even longer follow-up throughout adulthood are needed to determine whether the lack of association persists. We had no data on diagnoses from primary health care. This shortage was in part compensated for by use of triptan purchases as a proxy, since data on all reimbursed triptan purchases prescribed in both specialised (including all medical specialties) and primary health outpatient care was available. Paediatric triptan usage was limited when our study participants were children; however, it is estimated that $6 \%$ of 12 - to 17 -year-old migraineurs in Finland were treated with triptans in 2007, at a time when our youngest study participants were 17 years old (22). Moreover, as we used stringent criteria for migraine, we may have missed milder cases not seeking medical attention and thus undiagnosed, or cases diagnosed in primary health care but not treated with triptans, given that NSAIDs like ibuprofen and acetaminophen (paracetamol) are often used as first line therapy in paediatric migraine. We chose not to include prescription drugs other than triptans, however, to avoid including individuals prescribed NSAIDs for indications other than migraine. Therefore, our study likely represents an underestimate of the true prevalence. On the other hand, the stringent criteria increase the likelihood that cases in our study actually represent true cases. Supporting the validity of our outcome variable, a recent Finnish study on migraine in an occupational health setting ended up with similar migraine frequencies, despite using somewhat different methodology $(7.4 \%$ of women and $2.1 \%$ of men, as compared with $7.3 \%$ and $2.4 \%$ in our study) (48). We were unable to account for possible variation in diagnostic threshold across hospital district. Being a register study, the diagnoses were not verified from medical records. Finally, the validity of the parental migraine covariate was restricted since it was not complemented with triptan purchases, and the follow-up time for parents was not accounted for.

This large, nationwide register-based study found no evidence for an association between preterm birth and subsequent risk of migraine up until young adulthood. The association between maternal smoking during pregnancy and subsequent migraine warrants attention and further study, given that prenatal smoking is an actionable lifestyle risk factor.

\section{Clinical implications}

- Preterm birth, across the entire gestational age range, was not a risk factor for subsequent migraine up until young adulthood.

- The results are encouraging for preterm individuals and their families, who face several other health challenges.

- Maternal smoking during pregnancy was associated with an increased risk of migraine in the offspring - an observation warranting attention and further study, given that prenatal smoking is an actionable lifestyle risk factor. 


\section{Declaration of conflicting interests}

The authors declared no potential conflicts of interest with respect to the research, authorship, and/or publication of this article.

\section{Funding}

The authors disclosed receipt of the following financial support for the research, authorship, and/or publication of this article: Supported by Academy of Finland (Grants 274794 and 315680 to EK); the European Commission (Horizon2020 award 733280 RECAP Research on Children and Adults Born Preterm); Norface DIAL project 462-16-040 Premlife Life Course Dynamics after Preterm Birth Protective Factors for Social and Educational Transitions, Health and Prosperity; Diabetes Research Foundation; Finnish Foundation for Pediatric Research; Juho Vainio Foundation; Novo Nordisk Foundation; Signe and Ane Gyllenberg Foundation; Sigrid Jusélius Foundation; Yrjö Jahnsson Foundation. SS-K received a grant from HUS Helsinki University Hospital and The Wilhelm and Else Stockmann Foundation. KAIE was partly supported by funding from the European Union's Horizon 2020 Research and Innovation Program RECAP Preterm Project (grant no. 733280) and has received a grant from the Liaison Committee of St. Olavs Hospital and the Norwegian University of Science and Technology. The sponsors had no role in the planning or execution of any part of the study.

\section{ORCID iD}

Sonja Strang-Karlsson (D) https://orcid.org/0000-0002-85942077

\section{References}

1. Chawanpaiboon S, Vogel JP, Moller AB, et al. Global, regional, and national estimates of levels of preterm birth in 2014: A systematic review and modelling analysis. Lancet Glob Health 2019; 7: e37-e46.

2. Allotey J, Zamora J, Cheong-See F, et al. Cognitive, motor, behavioural and academic performances of children born preterm: A meta-analysis and systematic review involving 64061 children. BJOG 2018; 125: 16-25.

3. Pyhälä R, Wolford E, Kautiainen H, et al. Self-reported mental health problems among adults born preterm: A meta-analysis. Pediatrics 2017; 139: e20162690.

4. Hovi P, Andersson S, Eriksson JG, et al. Glucose regulation in young adults with very low birth weight. $N$ Engl J Med 2007; 356: 2053-2063.

5. Sipola-Leppänen $M$, Vääräsmäki $M$, Tikanmäki, et al. Cardiometabolic risk factors in young adults who were born preterm. Am J Epidemiol 2015; 181: 861-873.

6. Crump C, Howell EA, Stroustrup A, et al. Association of preterm birth with risk of ischemic heart disease in adulthood. JAMA Pediatr 2019; 173: 736-743.

7. Ueda $\mathrm{P}$, Cnattingius S, Stephansson $\mathrm{O}$, et al. Cerebrovascular and ischemic heart disease in young adults born preterm: A population-based Swedish cohort study. Eur J Epidemiol 2014; 29: 253-260.
8. Koupil I, Leon DA and Lithell HO. Length of gestation is associated with mortality from cerebrovascular disease. J Epidemiol Community Health 2005; 25: 473-474.

9. Kajantie E, Osmond C and Eriksson JG. Coronary heart disease and stroke in adults born preterm - the Helsinki Birth Cohort study. Paediatr Perinat Epidemiol 2015; 29: 515-519.

10. Adelborg K, Szépligeti SK, Holland-Bill L, et al. Migraine and risk of cardiovascular diseases: Danish population based matched cohort study. BMJ 2018; 360: $\mathrm{k} 96$.

11. Skajaa N, Szépligeti SK, Xue F, et al. Pregnancy, birth, neonatal, and postnatal neurological outcomes after pregnancy with migraine. Headache 2019; 59: 869-879.

12. Pisanu C, Lundin E, Preisig M, et al. Major depression subtypes are differentially associated with migraine subtype, prevalence and severity. Cephalalgia 2020; 40: 347-356.

13. Stewart WF, Roy J and Lipton RB. Migraine prevalence, socioeconomic status, and social causation. Neurology 2013; 81: 948-955.

14. Chen HM, Chen SF, Chen YH, et al. Increased risk of adverse pregnancy outcomes for women with migraines: A nationwide population-based study. Cephalalgia 2010; 30: 433-438.

15. Mortensen LH. Socioeconomic inequality in birth weight and gestational age in Denmark 1996-2007: Using a family-based approach to explore alternative explanations. Soc Sci Med 2013; 76: 1-7.

16. Hu J, Li Y, Zhang B, et al. Impact of the 2017 ACC/ AHA guideline for high blood pressure on evaluating gestational hypertension - associated risks for newborns and mothers. Circ Res 2019; 125: 184-194.

17. Ream MA and Lehwald L. Neurologic consequences of preterm birth. Curr Neurol Neurosci Rep 2018: 18: 48.

18. Alenius S, Kajantie E, Sund R, et al. The missing siblings of infants born preterm. Pediatrics 2018; 141: e20171354.

19. Furu K, Wettermark B, Andersen M, et al. The Nordic countries as a cohort for pharmacoepidemiological research. Basic Clin Pharmacol Toxicol 2010; 106: 86-94.

20. Rikala M, Hartikainen S, Sulkava R, et al. Validity of the Finnish Prescription Register for measuring psychotropic drug exposures among elderly Finns: A population-based intervention study. Drugs Aging 2010; 27: 337-349.

21. Haukka J, Suvisaari J, Tuulio-Henriksson A, et al. High concordance between self-reported medication and official prescription database information. Eur $J$ Clin Pharmacol 2007; 63: 1069-1074.

22. Lindkvist J, Airaksinen M, Kaukonen AM, et al. Evolution of paediatric off-label use after new significant medicines become available for adults: A study on triptans in Finnish children 1994-2007. Br J Clin Pharmacol 2011; 71: 929-935.

23. Sund R. Quality of the Finnish Hospital Discharge Register: A systematic review. Scand J Public Health 2012; 40: 505-515.

24. World Health Organization (WHO). Preterm birth, fact sheets, 19 February 2018, https://www.who.int/news- 
room/fact-sheets/detail/preterm-birth (accessed 14 March 2020).

25. Fleischman AR, Oinuma $M$ and Clark SL. Rethinking the definition of "term pregnancy". Obstet Gynecol 2010; 116: 136-139.

26. Victor TW, Hu X, Campbell JC, et al. Migraine prevalence by age and sex in the United States: A life-span study. Cephalalgia 2010; 30: 1065-1072.

27. Sankilampi U, Hannila ML, Saari A, et al. New population-based references for birth weight, length, and head circumference in singletons and twins from 23 to 43 gestation weeks. Ann Med 2013; 45: 446-454.

28. Therneau TM. A package for survival analysis in $\mathrm{S}$, https://CRAN.R-project.org/package $=$ survival $\quad(2014$, accessed March, 2020).

29. Paulsson L, Ekberg E, Nilner M, et al. Mandibular function, temporomandibular disorders, and headache in prematurely born children. Acta Odontol Scand 2009; 67: 30-37.

30. Fabbri CE, Barbieri MA, Silva AM, et al. Maternal smoking during pregnancy and primary headache in school-aged children: A cohort study. Cephalalgia 2012; 32: 317-327.

31. Maneyapanda SB and Venkatasubramanian A. Relationship between significant perinatal events and migraine severity. Pediatrics 2005; 116: e555-558.

32. Tavasoli A, Aghamohammadpoor $\mathbf{M}$ and Taghibeigi $\mathbf{M}$. Migraine and tension-type headache in children and adolescents presenting to neurology clinics. Iran J Pediatr 2013; 23: 536-540.

33. Børte S, Winsvold BS, Stensland SØ, et al. The effect of foetal growth restriction on the development of migraine and tension-type headache in adulthood. The HUNT Study. PLoS One 2017; 12: e0175908.

34. Waldie KE, Thompson JM, Mia Y, et al. Risk factors for migraine and tension-type headache in 11 year old children. J Headache Pain 2014; 15: 60.

35. Vetvik KG and MacGregor EA. Sex differences in the epidemiology, clinical features, and pathophysiology of migraine. Lancet Neurol 2017; 16: 76-87.

36. Ekblad M, Korkeila J and Lehtonen L. Smoking during pregnancy affects foetal brain development. Acta Paediatr 2015; 104: 12-18.

37. Arruda MA, Guidetti V, Galli F, et al. Prenatal exposure to tobacco and alcohol are associated with chronic daily headaches at childhood. Arq Neuropsiquiatr 2011; 69: 27-33.

38. Gormley P, Anttila V, Winsvold BS, et al. Meta-analysis of 375,000 individuals identifies 38 susceptibility loci for migraine. Nat Genet 2016; 48: 856-866.

39. Børte S, Zwart JA, Stensland SØ, et al. Parental migraine in relation to migraine in offspring: Family linkage analyses from the HUNT Study. Cephalalgia 2019; 39: 854-862.

40. Schwedt TJ. Chronic migraine. BMJ 2014; 348: g1416.

41. Hagen K, Einarsen C, Zwart JA, et al. The co-occurrence of headache and musculoskeletal symptoms amongst 51 050 adults in Norway. Eur J Neurol 2002; 9: 527-533.

42. Luedtke K, Starke W and May A. Musculoskeletal dysfunction in migraine patients. Cephalalgia 2018; 38: 865-875.

43. Iversen JM, Indredavik MS, Evensen KA, et al. Selfreported chronic pain in young adults with a low birth weight. Clin J Pain 2017; 33: 348-355.

44. Iversen JM, Hoftun GB, Romundstad PR, et al. Adolescent chronic pain and association to perinatal factors: Linkage of Birth Registry data with the YoungHUNT Study. Eur J Pain 2015; 19: 567-575.

45. Evensen KAI, Tikanmäki M, Heinonen K, et al. Musculoskeletal pain in adults born preterm: Evidence from two birth cohort studies. Eur J Pain 2019; 23: 461-471.

46. Official Statistics of Finland. Country of origin and language by age and sex, 1990-2014 (in Finnish, http:// pxnet2.stat.fi/PXWeb/pxweb/en/StatFin/StatFin_vrm _vaerak/statfin_vaerak_pxt_11ra.px/table/tableView Layout1/ (14 March 2020).

47. Global Burden of Disease 2016 Neurology Collaborators. Global, regional, and national burden of neurological disorders, 1990-2016: A systematic analysis for the Global Burden of Disease Study 2016. Lancet Neurol 2019; 18: 459-480.

48. Korolainen MA, Kurki S, Lassenius MI, et al. Burden of migraine in Finland: Health care resource use, sick-leaves and comorbidities in occupational health care. J Headache Pain 2019; 20: 13.

49. Alenius S, Kajantie E, Sund R, et al. Out-of-home care placements of children and adolescents born preterm: A register-based cohort study. Paediatr Perinat Epidemiol 2020; 34: 38-47. 